\title{
Solving Generalized Wave and Heat Equations Using Linear Canonical Transform and Sampling Formulae
}

\author{
Mawardi Bahri ${ }^{1}$ and Ryuichi Ashino $\mathbb{D}^{2}$ \\ ${ }^{1}$ Department of Mathematics, Hasanuddin University, Makassar 90245, Indonesia \\ ${ }^{2}$ Division of Mathematical Sciences, Osaka Kyoiku University, Osaka 582-8582, Japan \\ Correspondence should be addressed to Ryuichi Ashino; ashino@cc.osaka-kyoiku.ac.jp
}

Received 14 June 2020; Revised 16 August 2020; Accepted 28 September 2020; Published 23 October 2020

Academic Editor: Kunquan Lan

Copyright (c) 2020 Mawardi Bahri and Ryuichi Ashino. This is an open access article distributed under the Creative Commons Attribution License, which permits unrestricted use, distribution, and reproduction in any medium, provided the original work is properly cited.

Several essential properties of the linear canonical transform (LCT) are provided. Some results related to the sampling theorem in the LCT domain are investigated. Generalized wave and heat equations on the real line are introduced, and their solutions are constructed using the sampling formulae. Some examples are presented to illustrate our results.

\section{Introduction}

The linear canonical transform (LCT) is one of nontrivial generalizations of the Fourier transform (FT). Recently, the LCT has been investigated extensively both in theory and applications. In the connection with the LCT theory, many interesting papers on this topic have been already available in the literature (see [1-5]). From the applied point of view, the LCT also has been widely used in various areas such as optics, radar system analysis, phase retrieval, pattern recognition, and many other applications (see [6-8]).

It is known that the wave and heat equations are important in mathematical physics, applied mathematics, and engineering. Common methods to solve these equations are the Fourier and Laplace transforms, which give their exact solutions. The other techniques to have the solutions of these equations are the variation iteration method and the domain decomposition method, which coresponds to the adomian decomposition method [9]. Recently, the fundamental solution of the generalized wave equation was obtained using the fractional Fourier transform (FrFT) by [10]. Since the LCT is a general form of the FrFT and also it is closely related to the FT, it is possible to extend wave and heat equations into the LCT domain. However, as far as we observe, up to now, the solution of generalized heat equation using the sampling formulae and the LCT has not yet been published in the literature.
Therefore, in the present article, we provide useful properties and examples of the LCT. We will consider the wave equation in the LCT domain, and we will explore the solution of this equation using the LCT as exact analytic tool for solutions. We find a relation that the solution of the wave equation using the FT and the FrFT is a special case of the solution of these generalized wave equation. We explicitly discuss a generalized version of the heat equation in the LCT domains. Based on the sampling formulae related to the LCT, we further derive its solution followed by using the LCT. We emphasize that our solutions are nontrivial generalizations of the solutions of the heat equation using the sampling formulae and FT methods. For implementations, we finally provide a few examples to illustrate our results.

The remnants of the article are organized as follows: Section 2 introduces definitions and examples of the LCT, which will be needed in the sequel. The useful properties of the LCT are presented in Section 3. Some results related to the sampling theorems are demonstrated in Section 4. A general form of wave equation in the LCT domain is provided in Section 5. A general form of heat equation in the LCT domain and its solution using the sampling formulae are presented in Section 6. Its solution using LCT is discussed in detail in Section 7. Finally, Section 8 concludes our article. 


\section{Linear Canonical Transform}

Stuart A. Collin [11] introduced the linear canonical transforms (LCTs) during the early 1970s in paraxial optics. Independently, Moshinsky and Quesne [12] studied LCTs in quantum mechanics. Recently, Wolf [13] discussed in detail the development of LCTs including several complex extensions of the LCTs.

Let us start with the definition of the several dimensional linear canonical transform:

Definition 1. (see $[12,13])$. For an arbitrary $2 N \times 2 N$ symplectic matrix

$$
\mathbf{E}=\left[\begin{array}{ll}
\mathbf{a} & \mathbf{b} \\
\mathbf{c} & \mathbf{d}
\end{array}\right], \quad \mathbf{E} \boldsymbol{\Omega} \mathbf{E}^{T}=\boldsymbol{\Omega}, \quad \boldsymbol{\Omega}=\left[\begin{array}{cc}
\mathbf{0} & \mathbf{1}_{N} \\
-\mathbf{1}_{N} & \mathbf{0}
\end{array}\right],
$$

the $N$-dimensional of the linear canonical transform (LCT) is then defined as

$$
L_{\mathbf{E}}\{f\}(\boldsymbol{\xi})=\int_{\mathbb{R}^{n}} f(\mathbf{t}) K_{\mathbf{E}}(\boldsymbol{\xi}, \mathbf{t}) d \mathbf{t},
$$

where

$$
K_{\mathbf{E}}(\boldsymbol{\xi}, \mathbf{t})=\frac{1}{\sqrt{(2 \pi i)^{N} \operatorname{det} \mathbf{b}}} e^{\frac{i}{2}\left(\boldsymbol{\xi}^{T} \mathbf{b}^{-1} \mathbf{d} \xi-\xi^{T} \mathbf{b}^{-1} \mathbf{t}+\frac{1}{2} \mathbf{t}^{T} \mathbf{b}^{-1} \mathbf{t}\right)},
$$

where $i^{2}=-1$. Here, $\mathbf{E}$ is invertible and its inverse is given by

$$
\mathbf{E}^{-1}=\left[\begin{array}{ll}
\mathbf{a} & \mathbf{b} \\
\mathbf{c} & \mathbf{d}
\end{array}\right]^{-1}=\boldsymbol{\Omega E}^{T} \boldsymbol{\Omega}^{T}=\left[\begin{array}{cc}
\mathbf{d}^{T} & -\mathbf{b}^{T} \\
-\mathbf{c}^{T} & \mathbf{a}^{T}
\end{array}\right] .
$$

Secondly, let us introduce the inversion formula. Because of

$$
K_{\mathrm{E}}(\boldsymbol{\xi}, \mathbf{t})^{\stackrel{\AA}{\mathrm{a}}}=K_{\mathrm{E}^{-1}}(\mathbf{t}, \boldsymbol{\xi}),
$$

then, inversion formula of (2) has the following form:

$$
f(\mathbf{t})=\int_{\mathbb{R}^{n}} L_{\mathbf{E}}\{f\}(\boldsymbol{\xi}) K_{\mathbf{E}^{-1}}(\mathbf{t}, \boldsymbol{\xi}) d \boldsymbol{\xi}
$$

To solve the generalized wave and heat equations, we will only deal with the case that $N=1$. When $N=1$, Definition 1 mentioned above becomes

$$
L_{E}\{f\}(\xi)=F_{E}(\xi)=\left\{\begin{array}{l}
\int_{\mathbb{R}} f(t) K_{E}(\xi, t) d t, b \neq 0, \\
\sqrt{d} e^{i(c d / 2) \xi^{2}} f(d \xi), b=0,
\end{array}\right.
$$

where $K_{E}(\xi, t)$ is so-called kernel of the LCT given by

$$
K_{E}(\xi, t)=\frac{1}{\sqrt{2 \pi b i}} e^{1 / 2\left((a / b) t^{2}-(2 / b) t \xi+(d / b) \xi^{2}\right)},
$$

and the matrix parameter $E=\left[\begin{array}{ll}a & b \\ c & d\end{array}\right]$ satisfies $\operatorname{det}(E)=a d$ $-b c=1$, that is, $E \in S l(2, \mathbb{R})$, which denotes the special linear group over $\mathbb{R}$.
Based on (5), the LCT kernel (8) satisfies

$$
K_{E^{-1}}(t, \xi)=K_{E}(\xi, t)^{\star}=\frac{1}{\sqrt{-2 \pi b i}} e^{-i / 2\left((a / b) t^{2}-(2 / b) t \xi+(d / b) \xi^{2}\right)}
$$

where $f^{\star}$ stands for the complex conjugation of $f$. Equation (7) implies that for $b=0$, the LCT of a signal is essentially a chirp multiplication. Therefore, we only consider the case of $b \neq 0$ in this work. In the special case where $E=$ $\left[\begin{array}{cc}0 & 1 \\ -1 & 0\end{array}\right]$, the LCT mentioned above reduces to the Fourier transform (FT), that is,

$$
L_{E}\{f(t)\}(\xi)=\frac{1}{\sqrt{i}} \mathscr{F}\{f(t)\}(\xi)
$$

Here, the FT and its inverse are defined by (see [14-16])

$$
\begin{aligned}
\mathscr{F}\{f(t)\}(\xi) & =\frac{1}{\sqrt{2 \pi}} \int_{\mathbb{R}} f(t) e^{-i t \xi} d t, \quad f(t) \\
& =\frac{1}{\sqrt{2 \pi}} \int_{\mathbb{R}} \mathscr{F}\{f(t)\}(\xi) e^{i t \xi} d \xi
\end{aligned}
$$

provided that the integral exists. The connection between the FT and LCT is given by

$$
L_{E}\{f(t)\}(\xi)=\frac{1}{\sqrt{i b}} e^{(i d / 2 b) \xi^{2}} \mathscr{F}\left\{e^{(i a / 2 b) t^{2}} f\right\}\left(\frac{\xi}{b}\right) .
$$

An application of (9), we get the inverse transform of the LCT, that is,

$$
\begin{aligned}
L_{E^{-1}}\left[L_{E}\{f\}\right](t) & =f(t)=\lim _{l \rightarrow \infty} \int_{-l}^{l} L_{E}\{f\}(\xi) K_{E^{-1}}(t, \xi) d \xi \\
& =\int_{\mathbb{R}} L_{E}\{f\}(\xi) K_{E^{-1}}(t, \xi) d \xi
\end{aligned}
$$

Or, equivalently,

$$
f(t)=\frac{1}{\sqrt{-2 \pi b i}} \int_{\mathbb{R}} L_{E}\{f\}(\xi) e^{-i / 2\left((a / b) t^{2}-(2 / b) t \xi+(d / b) \xi^{2}\right)} d \xi .
$$

Definition 2. For every $E=\left[\begin{array}{ll}a & b \\ c & d\end{array}\right] \in \operatorname{Sl}(2, \mathbb{C})$, the complex LCT is defined by

$$
L_{E}\{f\}(z)=\int_{\mathbb{R}} f(x) K_{E}(z, x) d x
$$


where

$$
K_{E}(z, x)=\frac{1}{\sqrt{2 \pi b i}} e^{i / 2\left((a / b) x^{2}-(2 / b) x z+(d / b) z^{2}\right)},
$$

provided $\operatorname{Re}(i a / b)<0$. For $a$ is a real number, $b$ must satisfy $-\pi<\arg b<0$. For an example, taking $a=2^{-1 / 2}=d, b=i$ $2^{-1 / 2}=c$, we obtain (compare to [17])

$$
K_{E}(z, x)=\pi^{-1 / 2} 2^{-1 / 4} e^{-1 / 2\left(x^{2}+z^{2}\right)+2^{1 / 2} x z} .
$$

The next, following [13], we have.

$$
\begin{aligned}
\text { Remark 3. When } E & =\left[\begin{array}{cc}
1 & -2 i t \\
0 & 1
\end{array}\right] \text {, we obtain } \\
K_{E}(\xi, x) & =\frac{1}{\sqrt{4 \pi t}} e^{-(x-\xi)^{2} / 4 t}, \quad t>0 .
\end{aligned}
$$

This form is often so-called heat kernel or Gauss's kernel.

Definition 4. The inversion formula of the complex LCT is defined as in $[13,17]$.

$$
\begin{aligned}
f(x) & =\int_{\mathbb{C}} L_{E}\{f\}(z) K_{E}^{-1}(x, z) d^{2} \mu\left(z, z^{\mathfrak{a}}\right), d^{2} \mu\left(z, z^{\AA}\right) \\
& =v_{m}\left(z, z^{\mathfrak{a}}\right) d(\operatorname{Re} z) d(\operatorname{Im} z),
\end{aligned}
$$

where

$v_{m}\left(z, z^{\mathfrak{a}}\right)=\sqrt{\frac{2}{\pi v}} e^{1 / 2 v\left(u z^{2}-2 z z^{\mathfrak{a}}+u^{\mathfrak{a}} z^{\mathfrak{a}^{2}}\right)}, \quad u=a^{\mathfrak{a}} d-b^{\mathfrak{a}} c, \quad v=2 \operatorname{Im} a b^{\mathfrak{a}}>0$.

Example 5. (Dirac delta function). The Dirac delta function $\delta(t)$ is a generalized function satisfying the following:

$$
\delta(t)=0 \text { fort } \neq 0, \delta(0)=\infty, \int_{\mathbb{R}} \delta(t) d t=1
$$

By applying (7) to $\delta(t)$, we obtain

$$
L_{E}\{\delta(t)\}(\xi)=\int_{\mathbb{R}} \delta(t) K_{E}(\xi, t) d t=K_{E}(\xi, 0)=\frac{1}{\sqrt{2 \pi b i}} e^{i d / 2 b \xi^{2}} .
$$

Example 6. (Gaussian function). The Gaussian function is defined by

$$
g(t)=e^{-k t^{2}}, \quad k>0 .
$$
[1])

The LCT of the Gaussian function $g(t)$ is given by (see

$$
L_{E}\left\{e^{-k t^{2}}\right\}(\xi)=\frac{1}{\sqrt{a+2 k b i}} e^{\left(c+2 k d i / 2(2 k b-a i) \xi^{2}\right.} .
$$

Let us introduce the convolution of two complex functions in the LCT domain and the relationship between the convolution and its LCT (see [5, 18-20]).

Definition 7. Let $f, g \in L^{1}(\mathbb{R})$. Then, the convolution operator associated with the LCT is given by

$$
(f * g)(x)=\int_{\mathbb{R}} e^{i(t-t-x) a} f(t) g(x-t) d t .
$$

The LCT of the convolution of $f$ and $g$ is given by

$$
\frac{1}{\sqrt{2 \pi b i}} e^{i\left(d \xi^{2} / 2 b\right)} L_{E}\{f * g\}(\xi)=L_{E}\{f\}(\xi) L_{E}\{g\}(\xi) .
$$

\section{Useful Properties of LCT}

The following Theorem 8 describes the LCT of the derivative of a function (see [21]). It is very useful to solve partial differential equations related to the LCT.

Theorem 8. Let $f(t) \in L^{1}(\mathbb{R})$. Assume that $L_{E}\left\{\left(d^{n} / d t^{n}\right) f(t)\right.$ \}$(\xi)$ exist. Then, we have

$$
L_{E}\left\{\frac{d^{n}}{d t^{n}} f(t)\right\}(\xi)=\left(-c \xi i+a \frac{d}{d \xi}\right)^{n} L_{E}\{f(t)\}(\xi) .
$$

Let us give a simple application of Theorem 8 .

Example 9. (Mexican hat wavelet). The Mexican hat wavelet is defined by (see [22])

$$
M(t)=\left(1-t^{2}\right) e^{-t^{2} / 2} .
$$

The alternative form of (28) is

$$
M(t)=-\frac{d^{2}}{d t^{2}} e^{-t^{2} / 2}
$$

The LCT of (28) is given by

$$
L_{E}\{M(t)\}(\xi)=-\frac{1}{\sqrt{a+b i}} e^{h \xi^{2}}\left(-i c a+6 h a^{2} \xi-\left(4 h i c a+c^{2}\right) \xi^{2}+4 h^{2} a^{2} \xi^{3}\right) .
$$

Let us give a sketch of the proof of (30) below.

By applying Theorem 8 with $n=2$ and Example 6 , we get

$$
\begin{aligned}
L_{E}\{M(t)\}(\xi) & \left.=-L_{E}\left\{\frac{d^{2}}{d t^{2}} e^{-t^{2} / 2}\right)\right\}(\xi) \\
& \left.=-\left(-c \xi i+a \frac{d}{d \xi}\right)^{2} L_{E}\left\{e^{-t^{2} / 2}\right)\right\}(\xi) \\
& =-\left((-c \xi i)^{2}-i c a \xi \frac{d}{d \xi}-i c a \frac{d}{d \xi} \xi+a^{2} \frac{d^{2}}{d \xi^{2}}\right) \frac{1}{\sqrt{a+b i}} e^{(c+d i / 2(b-a i)) \xi^{2}} .
\end{aligned}
$$


By algebraic simplifications, we obtain

$L_{E}\{M(t)\}(\xi)=-\frac{1}{\sqrt{a+b i}}\left(-c^{2} \xi^{2}-i c a \xi \frac{d}{d \xi}-i c a \frac{d}{d \xi} \xi+a^{2} \frac{d^{2}}{d \xi^{2}}\right) e^{1 / 2\left(\left((a c+b d) i-1 / a^{2}+b^{2}\right)\right) \xi^{2}}$.

Now, setting $h=1 / 2\left(\left((a c+b d) i-1 / a^{2}+b^{2}\right)\right)$, we get

$$
\begin{aligned}
L_{E}\{M(t)\}(\xi)= & -\frac{1}{\sqrt{a+b i}}\left(-c^{2} \xi^{2}-i c a \xi \frac{d}{d \xi}-i c a \frac{d}{d \xi} \xi+a^{2} \frac{d^{2}}{d \xi^{2}}\right) e^{h \xi^{2}} \\
= & -\frac{1}{\sqrt{a+b i}}\left(-c^{2} \xi^{2} e^{h \xi^{2}}-i c a \xi \frac{d}{d \xi} e^{h \xi^{2}}-i c a \frac{d}{d \xi} \xi e^{h \xi^{2}}+a^{2} \frac{d^{2}}{d \xi^{2}} e^{h \xi^{2}}\right) \\
= & -\frac{1}{\sqrt{a+b i}}\left(-c^{2} \xi^{2} e^{h \xi^{2}}-2 h i c a \xi^{2} e^{h \xi^{2}}-i c a\left(1+2 h \xi^{2}\right) e^{h \xi^{2}}\right. \\
& \left.+a^{2} \frac{d}{d \xi}\left(1+2 h \xi^{2}\right) e^{h \xi^{2}}\right) \\
= & -\frac{1}{\sqrt{a+b i}}\left(-c^{2} \xi^{2} e^{h \xi^{2}}-2 h i c a \xi^{2} e^{h \xi^{2}}-i c a\left(1+2 h \xi^{2}\right) e^{h \xi^{2}}\right. \\
& \left.+a^{2}\left(4 h \xi+\left(1+2 h \xi^{2}\right) 2 h \xi\right) e^{h \xi^{2}}\right) \\
= & -\frac{1}{\sqrt{a+b i}} e^{h \xi^{2}}\left(-c^{2} \xi^{2}-2 h i c a \xi^{2}-i c a-2 h i c a \xi^{2}\right. \\
& \left.+4 h a^{2} \xi+2 h a^{2} \xi+4 h^{2} a^{2} \xi^{3}\right) \\
= & -\frac{1}{\sqrt{a+b i}} e^{h \xi^{2}}\left(-i c a+6 h a^{2} \xi-\left(4 h i c a+c^{2}\right) \xi^{2}+4 h^{2} a^{2} \xi^{3}\right) .
\end{aligned}
$$

Definition 10 . The Schwartz space $\delta(\mathbb{R})$ of rapidly decaying functions is defined by a collection of complex valued functions such that

$$
\mathcal{S}=\left\{\psi(t) \in C^{\infty}(\mathbb{R}): \sup _{t \in \mathbb{R}}\left|t^{m} D^{n} \psi(t)\right|<\infty, \forall m, n \in \mathbb{N}\right\},
$$

where $D=d / d t$.

The generalization of the Schwartz space in the LCT domain is described in the following.

Definition 11. ([21]). The Schwartz space $\mathcal{S}_{E}(\mathbb{R})$ of rapidly decaying functions associated with the LCT is defined by a collection of complex valued functions such that

$$
\mathcal{S}_{E}=\left\{\psi(t) \in C^{\infty}(\mathbb{R}): \sup _{t \in \mathbb{R}}\left|t^{m} D_{E}^{n} \psi(t)\right|<\infty, \forall m, n \in \mathbb{N}\right\},
$$

where $D_{E}=d / d t-i(a / b) t$.

From the above definition, we obtain the following important result (see [21]).
Theorem 12. Let $K_{E}(\xi, t)$ be the LCT kernel and $\bar{D}_{E}=-d / d t$ $-i(a / b) t$. Then, we have

$$
D_{E}^{n} K_{E}(\xi, t)=\left(-i \frac{\xi}{b}\right)^{n} K_{E}(\xi, t), \forall n \in \mathbb{N}
$$

$$
L_{E}\left\{\bar{D}_{E}^{n} \psi(t)\right\}(\xi)=\left(-i \frac{\xi}{b}\right)^{n} L_{E}\{\psi(t)\}(\xi), \forall n \in \mathbb{N} \text { and } \psi \in \mathcal{S}_{E}
$$

\section{Sampling Theorem in LCT Domain}

The sampling theorem [23] states that a class of functions can be determined perfectly from samples taken at equal interval. In this part, we investigate some results of sampling theorem associated with the LCT. We first introduce the following result, which was investigated in $[24,25]$.

Theorem 13. Suppose that $f$ is band limited to $\Omega_{E}$ in the LCT domain. Then, for every $x$ and $b>0$, one has

$$
f(x)=\sum_{n=-\infty}^{n=\infty} e^{-i(a / 2 b) x^{2}} f(n T) e^{i(a / 2 b)(n T)^{2}} \operatorname{sinc}\left[\frac{\Omega_{E}(x-n T)}{b \pi}\right] .
$$

Here, $T=\pi b / \Omega_{E}$, and it is the sampling period.

This gives the following theorem which is very useful to obtain the solution of heat equation in the LCT domain.

Theorem 14. Under assumptions as in Theorem 13, we have

$$
\begin{aligned}
L_{E}\{f\}(\xi)= & H_{0}\left(\xi+\Omega_{E}\right)-H_{0}\left(\xi-\Omega_{E}\right) \\
& \cdot \frac{\pi \sqrt{b \pi}}{\Omega_{E} \sqrt{2 i}} e^{i(d / 2 b) \xi^{2}} \sum_{n=-\infty}^{n=\infty} f(n T) e^{i(a / 2 b)(n T)^{2}} e^{-i n T \xi / b},
\end{aligned}
$$

where the step function is defined by

$$
H_{0}\left(\xi+\Omega_{E}\right)-H_{0}\left(\xi-\Omega_{E}\right)=\left(\left\{\begin{array}{ll}
1 & \text { if } \Omega_{E}<\xi<\Omega_{E} \\
0 & \text { otherwise. }
\end{array}\right.\right.
$$

Proof. Substituting (37) into the LCT definition, it is not difficult to arrive at 


$$
\begin{aligned}
L_{E}\{f\}(\xi)= & \int_{\mathbb{R}} f(x) \frac{1}{\sqrt{2 \pi b i}} e^{i / 2\left((a / b) x^{2}-(2 / b) x \xi+(d / b) \xi^{2}\right)} d x \\
= & \int_{\mathbb{R}} \sum_{n=-\infty}^{n=\infty} f(n T) e^{i(a / 2 b)(n T)^{2}} \operatorname{sinc}\left[\frac{\Omega_{E}(x-n T)}{b \pi}\right] \\
& \cdot \frac{1}{\sqrt{2 \pi b i}} e^{i / 2\left(-(2 / b) x \xi+(d / b) \xi^{2}\right)} d x \\
= & \frac{1}{\sqrt{2 \pi b i}} e^{i(d / 2 b) \xi^{2}} \sum_{n=-\infty}^{n=\infty} f(n T) e^{i(a / 2 b)(n T)^{2}} \int_{\mathbb{R}} \operatorname{sinc} \\
& \cdot\left[\frac{\Omega_{E}(x-n T)}{b \pi}\right] e^{-i(x \xi / b)} d x \\
= & \frac{1}{\sqrt{2 \pi b i}} e^{i(d / 2 b) \xi^{2}} \sum_{n=-\infty}^{n=\infty} f(n T) e^{i(a / 2 b)(n T)^{2}} \int_{\mathbb{R}} \sin \\
& \cdot\left[\frac{\left.\left(\Omega_{E} / b \pi\right) x-\left(\Omega_{E} T / b \pi^{2}\right) n \pi\right]}{\left(\Omega_{E} / b \pi\right) x-\left(\Omega_{E} T / b \pi^{2}\right) n \pi}\right] e^{-i(x \xi / b)} d x \\
= & H_{0}\left(\xi+\Omega_{E}\right)-H_{0}\left(\xi-\Omega_{E}\right) e^{i(d / 2 b) \xi^{2}} \sum_{n=-\infty}^{n=\infty} f \\
& \cdot(n T) e^{i(a / 2 b)(n T)^{2}} \frac{b \pi}{\Omega_{E}} e^{-i n T \xi / b} \sqrt{\frac{\pi}{2 b i}} \\
= & H_{0}\left(\xi+\Omega_{E}\right)-H_{0}\left(\xi-\Omega_{E}\right) \\
& \cdot \frac{\pi \sqrt{b \pi}}{\Omega_{E} \sqrt{2 i}} e^{i(d / 2 b) \xi^{2}} \sum_{n=-\infty}^{n=\infty} f(n T) e^{i(a / 2 b)(n T)^{2}} e^{-i n T \xi / b} .
\end{aligned}
$$

This is the desired result.

\section{Generalized Wave Equations in Linear Canonical Transform Domain}

In the present section, we study how the LCT can be applied to solve partial differential equations. In particular, we are going to obtain the solution of generalized wave equation. In [26], the author have obtained the solution of wave equation using the LCT. Therefore, in this part, we propose a different way to obtain the solution of wave equations related to the LCT. We also provide a simple example to illustrate the result. Let us therefore introduce the generalized wave equation in the LCT domain.

Consider the following generalized one-dimensional wave equation associated with the LCT:

$$
\frac{\partial^{2} \phi(x, t)}{\partial t^{2}}=k^{2} \bar{D}_{E}^{2} \phi(x, t), \quad-\infty<x<\infty, \quad t>0,
$$

with the initial condition:

$$
\phi(x, 0)=f(x),\left.\quad \frac{\partial \phi(x, t)}{\partial t}\right|_{t=0}=g(x), \quad-\infty<x<\infty
$$

where $k$ is a positive constant and $f(x), g(x) \in L^{1}(\mathbb{R})$. Note that $\bar{D}_{E}=-d / d x-i(a / b) x$. The solution of the generalized one-dimensional wave equation mentioned above is given by

$$
\begin{aligned}
\phi(x, t)= & \operatorname{sgn}(b) \frac{e^{-i(a / 2 b) x^{2}}}{2} \times\left[f(x+k t) e^{i(a / 2 b)(x+k t)^{2}}\right. \\
& \left.+f(x-k t) e^{i(a / 2 b)(x-k t)^{2}}+\frac{1}{k} \int_{x-k t}^{x+k t} g(\xi) e^{i(a / 2 b) \xi^{2}} d \xi\right] .
\end{aligned}
$$

Note that, in the case when $E=\left[\begin{array}{cc}0 & 1 \\ -1 & 0\end{array}\right]$, the solution (43) becomes the well-known d'Alembert solution of the classical wave equation, that is,

$$
\begin{gathered}
\phi(x, t)=\frac{1}{2}\left[f(x+k t)+f(x-k t)+\frac{1}{k} \int_{x-k t}^{x+k t} g(\xi) d \xi\right] . \quad(44) \\
\text { When } E=\left[\begin{array}{cc}
\cos \alpha & \sin \alpha \\
-\sin \alpha & \cos \alpha
\end{array}\right],(43) \text { leads to } \\
\phi(x, t)=\operatorname{sgn}(\sin \alpha) \frac{e^{-i(\cot \alpha / 2) x^{2}}}{2}\left[f(x+k t) e^{i(\cot \alpha / 2)(x+k t)^{2}}\right. \\
\left.+f(x-k t) e^{i(\cot \alpha / 2)(x-k t)^{2}}+\frac{1}{k} \int_{x-k t}^{x+k t} g(\xi) e^{i(\cot \alpha / 2) \xi^{2}} d \xi\right],
\end{gathered}
$$

which is the solution of the generalized wave equation using the FrFT (compare to [10]). Therefore, (43) can be seen as follows:

For fixed $t$, applying the LCT on both sides of (41) and using Theorem 12, we obtain

$$
\frac{\partial^{2} \phi_{E}(\xi, t)}{\partial t^{2}}=k^{2}\left(-i \frac{\xi}{b}\right)^{2} \phi_{E}(\xi, t)
$$

where $\phi_{E}(\xi, t)=L_{E}\{\phi(x, t)\}$ is the LCT of $\phi(x, t)$ with respect to $x$ only. The solution of equation (46) takes the form:

$$
\phi_{E}(\xi, t)=P_{E}(\xi) e^{(i k / b) \xi t}+Q_{E}(\xi) e^{-(i k / b) \xi t} .
$$

Differentiating (47) with respect to $t$, we have

$$
\phi_{t_{E}}(\xi, t)=\frac{k}{b} \xi i P_{E}(\xi) e^{(i k / b) \xi t}-\frac{k}{b} \xi i Q_{E}(\xi) e^{-(i k / b) \xi t} .
$$

Now, we use the notation

$$
\begin{array}{r}
\left.F_{E}(\xi)=L_{E}\{f(x)\}(\xi)=L_{E}\{\phi(x, 0)\} \xi\right)=\phi_{E}(\xi, 0), \\
\left.G_{E}(\xi)=L_{E}\{g(x)\}(\xi)=L_{E}\left\{\phi_{t}(x, 0)\right\} \xi\right)=\phi_{t_{E}}(\xi, 0) .
\end{array}
$$

Consequently, we obtain

$$
\begin{gathered}
F_{E}(\xi)=\phi_{E}(\xi, 0)=P_{E}(\xi)+Q_{E}(\xi), \\
G_{E}(\xi)=\phi_{t_{E}}(\xi, 0)=\frac{k}{\xi} \xi i\left(P_{E}(\xi)-Q_{E}(\xi)\right) .
\end{gathered}
$$

From equations (51) and (52), we get

$$
\begin{aligned}
P_{E}(\xi) & =\frac{1}{2}\left(F_{E}(\xi)+\frac{b}{i k \xi} G_{E}(\xi)\right), \\
Q_{E}(\xi) & =\frac{1}{2}\left(F_{E}(\xi)-\frac{b}{i k \xi} G_{E}(\xi)\right) .
\end{aligned}
$$


Substituting (54) in (47) yields

$$
\begin{aligned}
\left.\phi_{E}(\xi, t)\right)= & \frac{1}{2} F_{E}(\xi)\left(e^{(i k / b) \xi t}+e^{-(i k / b) \xi t}\right) \\
& +\frac{b}{2 i k \xi} G_{E}(\xi)\left(e^{(i k / b) \xi t}-e^{-(i k / b) \xi t}\right) .
\end{aligned}
$$

Now, applying inverse transform of the LCT defined by (14) into both sides of (55), we immediately get

$$
\begin{aligned}
& \phi(x, t)=\frac{1}{\sqrt{-2 \pi b i}} \int_{\mathbb{R}}\left[\left(\frac{1}{2} F_{E}(\xi)\left(e^{(i k / b) \xi t}+e^{-(i k / b) \xi t}\right)\right.\right. \\
& \left.+\frac{b}{2 i k \xi} G_{E}(\xi)\left(e^{(i k / b) \xi t}-e^{-(i k / b) \xi t}\right)\right) \\
& \left.\times e^{-i / 2\left((a / b) x^{2}-(2 / b) x \xi+(d / b) \xi^{2}\right)}\right] d \xi \\
& =\frac{1}{\sqrt{-2 \pi b i}} \int_{\mathbb{R}}\left[\left(\frac{1}{2} \frac{1}{\sqrt{2 \pi b i}} \int_{\mathbb{R}} f(\varepsilon) e^{i / 2\left((a / b) \varepsilon^{2}-(2 / b) \varepsilon \xi+(d / b) \xi^{2}\right)} d \varepsilon\right.\right. \\
& \cdot\left(e^{(i k / b) \xi t}+e^{-(i k / b) \xi t}\right)+\frac{b}{2 i k \xi} \\
& \cdot \frac{1}{\sqrt{2 \pi b i}} \int_{\mathbb{R}} g(\varepsilon) e^{i / 2\left((a / b) \varepsilon^{2}-(2 / b) \varepsilon \xi+(d / b) \xi^{2}\right)} d \varepsilon \\
& \left.\left.\cdot\left(e^{(i k / b) \xi t}-e^{-(i k / b) \xi t}\right)\right) e^{-i / 2\left((a / b) x^{2}-(2 / b) x \xi+(d / b) \xi^{2}\right)}\right] d \xi \\
& =\frac{1}{\sqrt{-2 \pi b i}} \frac{1}{\sqrt{2 \pi b i}} \int_{\mathbb{R}}\left[\frac { 1 } { 2 } \left(\int_{\mathbb{R}} f(\varepsilon) e^{i / 2\left((a / b) \varepsilon^{2}-(2 / b) \varepsilon \xi+(d / b) \xi^{2}\right)} d \varepsilon\right.\right. \\
& \cdot\left(e^{(i k / b) \xi t}+e^{-(i k / b) \xi t}\right) \\
& +\frac{b}{2 i k \xi} \int_{\mathbb{R}} g(\varepsilon) e^{i / 2\left((a / b) \varepsilon^{2}-(2 / b) \varepsilon \xi+(d / b) \xi^{2}\right)} d \varepsilon \\
& \left.\left.\cdot\left(e^{(i k / b) \xi t}-e^{-(i k / b) \xi t}\right)\right) e^{-i / 2\left((a / b) x^{2}-(2 / b) x \xi+(d / b) \xi^{2}\right)}\right] d \xi \\
& =\frac{1}{2 \pi|b|} \int_{\mathbb{R}}\left[\frac{1}{2} \int_{\mathbb{R}} f(\varepsilon) e^{i / 2\left((a / b) \varepsilon^{2}-(2 / b) \varepsilon \xi\right)}\right. \\
& \cdot\left(e^{(i k / b) \xi t}+e^{-(i k / b) \xi t}\right) e^{-i / 2\left((a / b) x^{2}-(2 / b) x \xi\right)} d \varepsilon \\
& +\frac{b}{2 i k \xi} \int_{\mathbb{R}} g(\varepsilon) e^{i / 2\left((a / b) \varepsilon^{2}-(2 / b) \varepsilon \xi\right)} \\
& \left.\cdot\left(e^{(i k / b) \xi t}-e^{-(i k / b) \xi t}\right) e^{-i / 2\left((a / b) x^{2}-(2 / b) x \xi\right)} d \varepsilon\right] d \xi \\
& =\frac{e^{-i(a / 2 b) x^{2}}}{2 \pi|b|} \int_{\mathbb{R}}\left[\frac{1}{2} \int_{\mathbb{R}} f(\varepsilon) e^{i / 2\left((a / b) \varepsilon^{2}-(2 / b) \varepsilon \xi\right)}\right. \\
& \cdot\left(e^{(i k / b) \xi t}+e^{-(i k / b) \xi t}\right) e^{-i / 2(-(2 / b) x \xi)} d \varepsilon \\
& +\frac{b}{2 i k \xi} \int_{\mathbb{R}} g(\varepsilon) e^{i / 2\left((a / b) \varepsilon^{2}-(2 / b) \varepsilon \xi\right)} \\
& \left.\cdot\left(e^{(i k / b) \xi t}-e^{-(i k / b) \xi t}\right) e^{-i / 2(-(2 / b) x \xi)} d \varepsilon\right] d \xi \\
& =\frac{e^{-i(a / 2 b) x^{2}}}{2 \pi|b|} \int_{\mathbb{R}}\left[\frac{1}{2} \int_{\mathbb{R}} f(\varepsilon) e^{i(a / 2 b) \varepsilon^{2}} e^{-(i \varepsilon \xi / b)}\right. \\
& \cdot\left(e^{(i k / b) \xi t}+e^{-(i k / b) \xi t}\right) e^{-i / 2(-(2 / b) x \xi)} d \varepsilon \\
& +\frac{b}{2 i k \xi} \int_{\mathbb{R}} g(\varepsilon) e^{i(a / 2 b) \varepsilon^{2}} e^{-(i \varepsilon \xi / b)} \\
& \left.\cdot\left(e^{(i k / b) \xi t}-e^{-(i k / b) \xi t}\right) e^{-i / 2(-(2 / b) x \xi)} d \varepsilon\right] d \xi e^{i(a / 2 b) \varepsilon^{2}} e^{-(i \varepsilon \xi / b)} \text {. }
\end{aligned}
$$

Set $\overline{\bar{f}}(\varepsilon)=f(\varepsilon) e^{i(a / 2 b) \varepsilon^{2}}, \overline{\bar{g}}(\varepsilon)=g(\varepsilon) e^{i(a / 2 b) \varepsilon^{2}}$, and $v=\xi / b$, then we have

$$
\begin{aligned}
\phi(x, t)= & \frac{b e^{-i(a / 2 b) x^{2}}}{2 \sqrt{2 \pi}|b|} \int_{\mathbb{R}}\left[\frac{1}{\sqrt{2 \pi}} \int_{\mathbb{R}} \overline{\bar{f}}(\varepsilon) e^{-i v \varepsilon} d \varepsilon\right. \\
& \cdot\left(e^{i k v t}+e^{-i k v t}\right) e^{i x v}+\frac{1}{i k v} \\
& \left.\cdot \frac{1}{\sqrt{2 \pi}} \int_{\mathbb{R}} \overline{\bar{g}}(\varepsilon) e^{-i v \varepsilon} d \varepsilon\left(e^{i k v t}-e^{-i k v t}\right) e^{i x v}\right] d v .
\end{aligned}
$$

By using the FT definition (11), we immediately obtain

$$
\begin{aligned}
& \phi(x, t)=\frac{b}{2|b|} \frac{e^{-i(a / 2 b) x^{2}}}{\sqrt{2 \pi}} \int_{\mathbb{R}}\left[\mathscr{F}\{\overline{\bar{f}}\}(v)\left(e^{i v(x+k t)}+e^{i v(x-k t)}\right)\right. \\
& \left.+\frac{b}{i k v} \mathscr{F}\{\overline{\bar{g}}\}(v)\left(e^{i v(x+k t)}-e^{i v(x-k t)}\right)\right] d v \\
& =\frac{\operatorname{sgn}(b)}{2} \frac{e^{-i(a / 2 b) x^{2}}}{\sqrt{2 \pi}}[\sqrt{2 \pi}(\overline{\bar{f}}(x+k t)+\overline{\bar{f}}(x-k t)) \\
& \left.+\frac{1}{k} \int_{\mathbb{R}} \mathscr{F}\{\overline{\bar{g}}\}(v)\left(\int_{x-k t}^{x+k t} e^{i v \alpha} d \alpha\right) d v\right] \\
& =\frac{\operatorname{sgn}(b)}{2} \frac{e^{-i(a / 2 b) x^{2}}}{\sqrt{2 \pi}}\left[\sqrt { 2 \pi } \left(f(x+k t) e^{i(a / 2 b)(x+k t)^{2}}\right.\right. \\
& \left.+f(x-k t) e^{i(a / 2 b)(x-k t)^{2}}\right)+\frac{1}{k} \int_{x-k t}^{x+k t} \\
& \left.\cdot\left(\int_{\mathbb{R}} \mathscr{F}\{\overline{\bar{g}}\}(v) e^{i v \alpha} d v\right) d \alpha\right] \\
& =\frac{\operatorname{sgn}(b) e^{-i(a / 2 b) x^{2}}}{2}\left[f(x+k t) e^{i(a / 2 b)(x+k t)^{2}}\right. \\
& +f(x-k t) e^{i(a / 2 b)(x-k t)^{2}}+\frac{1}{k} \int_{x-k t}^{x+k t} \\
& \left.\cdot\left(\frac{1}{\sqrt{2 \pi}} \int_{\mathbb{R}} \mathscr{F}\{\overline{\bar{g}}\}(v) e^{i v \alpha} d v\right) d \alpha\right] \\
& =\frac{\operatorname{sgn}(b) e^{-i(a / 2 b) x^{2}}}{2}\left[f(x+k t) e^{i(a / 2 b)(x+k t)^{2}}\right. \\
& \left.+f(x-k t) e^{i(a / 2 b)(x-k t)^{2}}+\frac{1}{k}\left(\int_{x-k t}^{x+k t} \overline{\bar{g}}(\alpha) d \alpha\right)\right] \\
& =\frac{\operatorname{sgn}(b) e^{-i(a / 2 b) x^{2}}}{2}\left[f(x+k t) e^{i(a / 2 b)(x+k t)^{2}}\right. \\
& \left.+f(x-k t) e^{i(a / 2 b)(x-k t)^{2}}+\frac{1}{k}\left(\int_{x-k t}^{x+k t} g(\alpha) e^{i(a / 2 b) \alpha^{2}} d \alpha\right)\right],
\end{aligned}
$$

as claimed in (43). For different values of (43) is shown in Figures 1 and 2.

For an illustration, we provide the following simple example. 
Example 5.1 Let us consider the following initial wave equation:

$$
\left\{\begin{array}{l}
\frac{\partial^{2} \phi(x, t)}{\partial t^{2}}=0.25 \bar{D}_{E}^{2} \phi(x, t), \quad-\infty<x<\infty, \quad t>0 \\
\phi(x, 0)=e^{-(1 / 2) x^{2}},\left.\quad \frac{\partial \phi(x, t)}{\partial t}\right|_{t=0}=e^{-(1 / 2) x^{2}} .
\end{array}\right.
$$

According to (43), we get the solution in the form:

$$
\begin{aligned}
\phi(x, t)= & \frac{\operatorname{sgn}(b)}{2} e^{-i(a / 2 b) x^{2}}\left[e^{-1 / 2(x+0.5 t)^{2}} e^{i / 2(x+0.5 t)^{2}}\right. \\
& +e^{-1 / 2(x-0.5)^{2}} e^{i / 2(x-0.5 t)^{2}} \\
& \left.+2 \int_{x-0.5 t}^{x+0.5 t} e^{-(1 / 2) \xi^{2}} e^{i(a / 2 b) \xi^{2}} d \xi\right]
\end{aligned}
$$

On the other hand, for $a=b=1$, we get

$$
\begin{aligned}
\int_{x-0.5 t}^{x+0.5 t} e^{-(1 / 2) \xi^{2}} e^{i(a / 2 b) \xi^{2}} d \xi & =\int_{x-0.5 t}^{x+0.5 t} e^{-1 / 2(1-i) \xi^{2}} d \xi \\
& =\int_{x-0.5 t}^{x+0.5 t} e^{-(\sqrt{(1 / 2)(1-i) \xi})^{2}} d \xi .
\end{aligned}
$$

Setting $u=\sqrt{(1 / 2)(1-i) \xi}$, then

$$
\begin{aligned}
\int_{x-0.5 t}^{x+0.5 t} e^{-(1 / 2) \xi^{2}} e^{i(a / 2 b) \xi^{2}} d \xi= & \frac{1}{\sqrt{1 / 2(1-i)}} \int_{\sqrt{(1 / 2)(1-i)}(x-0.5 t)}^{\sqrt{(1 / 2)(1-i)}(x+0.5 t)} e^{-u^{2}} d u \\
= & \frac{2}{\sqrt{2-2 i}} \int_{1 / 4 \sqrt{(2-2 i)(2 x-t)}}^{1 / 4 \sqrt{(2-2 i)(2 x+t)}} e^{-u^{2}} d u \\
= & \frac{\sqrt{\pi}}{\sqrt{2-2 i}}\left(\frac{2}{\sqrt{\pi}} \int_{1 / 4 \sqrt{(2-2 i)}(2 x-t)}^{0} e^{-u^{2}} d u\right. \\
& \left.+\frac{2}{\sqrt{\pi}} \int_{0}^{1 / 4 \sqrt{(2-2 i)}(2 x+t)} e^{-u^{2}} d u\right) \\
= & \frac{\sqrt{\pi}}{\sqrt{2-2 i}}\left(\frac{2}{\sqrt{\pi}} \int_{0}^{1 / 4 \sqrt{(2-2 i)}(t-2 x)} e^{-u^{2}} d u\right. \\
& \left.+\frac{2}{\sqrt{\pi}} \int_{0}^{1 / 4} \sqrt{(2-2 i)(2 x+t)} e^{-u^{2}} d u\right) \\
= & \frac{\sqrt{\pi}}{\sqrt{2-2 i}}\left(\operatorname{erf}\left(\frac{1}{4} \sqrt{2-2 i}(-2 x+t)\right)\right. \\
& \left.+\operatorname{erf}\left(\frac{1}{4} \sqrt{2-2 i}(2 x+t)\right)\right)
\end{aligned}
$$

It means that equation (60) takes the form

$$
\begin{aligned}
\phi(x, t)= & \frac{e^{-(i / 2) x^{2}}}{2}\left[e^{-1 / 2(x+0.5 t)^{2}} e^{i / 2(x+0.5 t)^{2}}\right. \\
& +e^{-1 / 2(x-0.5 t)^{2}} e^{i / 2(x-0.5 t)^{2}} \\
& +\frac{2 \sqrt{\pi}}{\sqrt{2-2 i}}\left(\operatorname{erf}\left(\frac{1}{4} \sqrt{2-2 i}(-2 x+t)\right)\right. \\
& \left.\left.+\operatorname{erf}\left(\frac{1}{4} \sqrt{2-2 i}(2 x+t)\right)\right)\right],
\end{aligned}
$$

where the error function is defined by

$$
\operatorname{erf}(x)=\frac{2}{\sqrt{\pi}} \int_{0}^{x} e^{-t^{2}} d t, \text { for all } x
$$

In particular, if $g(x)=0$, the above expression becomes

$$
\phi(x, t)=\frac{e^{i\left(t^{2} / 8\right)}}{2}\left[e^{-1 / 2(x+0.5 t)^{2}} e^{i 0.5 x t}+e^{-1 / 2(x-0.5 t)^{2}} e^{-i 0.5 x t}\right] .
$$

\section{Solution of Generalized Heat Equation Using Sampling Formulae}

Let us consider the following generalized one-dimensional heat equation associated with the LCT:

$$
\frac{\partial \varphi(x, t)}{\partial t}=k^{2} \bar{D}_{E}^{2} \varphi(x, t), \quad-\infty<x<\infty, \quad t>0,
$$

with the initial condition:

$$
\varphi(x, 0)=f(x)
$$

where $k \neq 0$ is a constant and $\bar{D}_{E}=-d / d x-i(a / b) x$.

Theorem 15. Assume that $f(x)$ in (67) is band limited to $\Omega_{E}$ in the LCT domain. Then, the solution of (66) is given by

$$
\begin{aligned}
\varphi(x, t)= & \frac{\pi}{\Omega_{E}} e^{-i(a / 2 b) x^{2}} \sum_{n=-\infty}^{n=\infty} f(n T) e^{i(a / 2 b)(n T)^{2}} \int_{-\Omega_{E}}^{\Omega_{E}} e^{-\left(k^{2} / b^{2}\right) \xi^{2} t} \cos \\
& \cdot\left(\xi\left(\frac{n T}{b}-\frac{x \xi}{b}\right)\right) d \xi .
\end{aligned}
$$

Proof. Applying the LCT to both sides of (66) yields

$$
\frac{\partial \varphi_{E}(\xi, t)}{\partial t}=-\frac{k^{2}}{b^{2}} \xi^{2} \varphi_{E}(\xi, t)
$$

This gives

$$
\varphi_{E}(\xi, t)=F_{E}(\xi) e^{-\left(k^{2} / b^{2}\right) \xi^{2} t}
$$

where $F_{E}(\xi)$ is defined by (49). Taking the inverse transform of the LCT in (70), it is easy to obtain 


$$
\begin{aligned}
\varphi(x, t) & =L_{E^{-1}}\left[F_{E}(\xi) e^{-\left(k^{2} / b^{2}\right) \xi^{2} t}\right] \\
& =\frac{1}{\sqrt{-2 \pi b i}} \int_{\mathbb{R}} F_{E}(\xi) e^{-\left(k^{2} / b^{2}\right) \xi^{2} t} e^{i / 2\left((a / b) x^{2}-(2 / b) x \xi+(d / b) \xi^{2}\right)} d \xi .
\end{aligned}
$$

Because $f(x)$ is band limited to $\Omega_{E}$, then $L_{E}\{f\}(\xi)=0$ for $|\xi|>\Omega_{E}$. Applying this fact and then substituting (38) in (71), we obtain

$$
\begin{aligned}
\varphi(x, t) & =\frac{1}{\sqrt{-2 \pi b i}} e^{-i(a / 2 b) x^{2}} \int_{\mathbb{R}} e^{-i(d / 2 b) \xi^{2}} F_{E}(\xi) e^{-\left(k^{2} / b^{2}\right) \xi^{2} t} e^{-i(x \xi / b)} d \xi \\
& =\frac{1}{\sqrt{-2 \pi b i}} e^{-i(a / 2 b) x^{2}} \int_{-\Omega_{E}}^{\Omega_{E}} e^{-i(d / 2 b) \xi^{2}} e^{i(d / 2 b) \xi^{2}} \sum_{n=-\infty}^{n=\infty} f(n T) e^{i(a / 2 b)(n T)^{2}} \frac{\pi \sqrt{b \pi}}{\Omega_{E} \sqrt{2 i}} e^{-i(n T \xi / b)} e^{-\left(k^{2} / b^{2}\right) \xi^{2} t} e^{-i(x \xi / b)} d \xi \\
& =\frac{1}{\sqrt{-2 \pi b i}} e^{-i(a / 2 b) x^{2}} \int_{-\Omega_{E}}^{\Omega_{E}} \sum_{n=-\infty}^{n=\infty} f(n T) e^{i(a / 2 b)(n T)^{2}} \frac{\pi \sqrt{b \pi}}{\Omega_{E} \sqrt{2 i}} e^{-i(n T \xi / b)} e^{-\left(k^{2} / b^{2}\right) \xi^{2} t} e^{-i(x \xi / b)} d \xi \\
& =\frac{1}{\sqrt{-2 \pi b i}} e^{-i(a / 2 b) x^{2}} \sum_{n=-\infty}^{n=\infty} f(n T) e^{i(a / 2 b)(n T)^{2}} \frac{\pi \sqrt{b \pi}}{\Omega_{E} \sqrt{2 i}} \int_{-\Omega_{E}}^{\Omega_{E}} e^{-\left(k^{2} / b^{2}\right) \xi^{2} t} e^{-i \xi((n T / b)-(x \xi / b))} d \xi \\
& =\frac{\pi}{2 \Omega_{E}} e^{-i(a / 2 b) x^{2}} \sum_{n=-\infty}^{n=\infty} f(n T) e^{i(a / 2 b)(n T)^{2}} \int_{-\Omega_{E}}^{\Omega_{E}} e^{-\left(k^{2} / b^{2}\right) \xi^{2} t} e^{-i \xi((n T / b)-(x \xi / b))} d \xi \\
& =\frac{\pi}{\Omega_{E}} e^{-i(a / 2 b) x^{2}} \sum_{n=-\infty}^{n=\infty} f(n T) e^{i(a / 2 b)(n T)^{2}} \int_{-\Omega_{E}}^{\Omega_{E}} e^{-\left(k^{2} / b^{2}\right) \xi^{2} t} \cos \left(\xi\left(\frac{n T}{b}-\frac{x \xi}{b}\right)\right) d \xi .
\end{aligned}
$$

The proof is complete.

\section{Solution of Generalized Heat Equation Using LCT}

The purpose of this part is to solve (66) using the LCT. We prove the following result.
Theorem 16. Assume that $f(x)$ in (67) belong $L^{1}(\mathbb{R})$. Then, the solution of (66) is given by

$$
\varphi(x, t)=\frac{1}{\sqrt{-4 \pi k^{2} t}} \int_{\mathbb{R}} e^{i u(u-x) a / b} f(u) e^{1 / 2\left(-b^{2} c-2 a k^{2} i t / 2 k^{2} b t\right)(x-u)^{2}} d u .
$$

Proof. By using the inverse transform of the LCT (14), we have

$$
\begin{aligned}
& L_{E^{-1}}\left[e^{-\left(k^{2} / b^{2}\right) \xi^{2} t}\right]=\frac{1}{\sqrt{-2 \pi b i}} \int_{\mathbb{R}} e^{-\left(k^{2} / b^{2}\right) \xi^{2} t} e^{-i / 2\left((a / b) x^{2}-(2 / b) x \xi+(d / b) \xi^{2}\right)} d \xi \\
& =\frac{e^{-(i a / 2 b) x^{2}}}{\sqrt{-2 \pi b i}} \int_{\mathbb{R}} e^{\left(-\left(k^{2} / b^{2}\right) t-d i / 2 b\right) \xi^{2}} e^{(-i \xi x / b)} d \xi \\
& =\frac{e^{-(i a / 2 b) x^{2}}}{\sqrt{-2 \pi b i}} \int_{\mathbb{R}} e^{\left(2 k^{2} t-i d b / 2 b^{2}\right)\left(\xi^{2}-2 i b \xi x / 2 k^{2} t-i d b\right)} d \xi \\
& =\frac{e^{-(i a / 2 b) x^{2}}}{\sqrt{-2 \pi b i}} \int_{\mathbb{R}} e^{\left(2 k^{2} t-i d b / 2 b^{2}\right)\left(\left(\xi^{2}-2 i b \xi x / 2 k^{2} t-i d b\right)+\left(i b x / 2 k^{2} t-i d b\right)^{2}-\left(i b x / 2 k^{2} t-i d b\right)^{2}\right)} d \xi \\
& =\frac{e^{-(i a / 2 b) x^{2}}}{\sqrt{-2 \pi b i}} e^{\left(2 k^{2} t-i d b / 2 b^{2}\right)\left(-\left(i b x / 2 k^{2} t-d b i\right)^{2}\right)} \int_{\mathbb{R}} e^{\left(2 k^{2} t-d b i / 2 b^{2}\right)\left(\left(\xi^{2}-2 i b \xi x / 2 k^{2} t-d b i\right)+\left(i b x / 2 k^{2} t-i d b\right)^{2}\right)} d \xi \\
& =\frac{e^{-(i a / 2 b) x^{2}}}{\sqrt{-2 \pi b i}} e^{x^{2} / 4 k^{2} t-2 d b i} \int_{\mathbb{R}} e^{-\left(d b i-2 k^{2} t / 2 b^{2}\right)\left(\xi-i b x / 2 k^{2} t-i d b\right)^{2}} d \xi .
\end{aligned}
$$




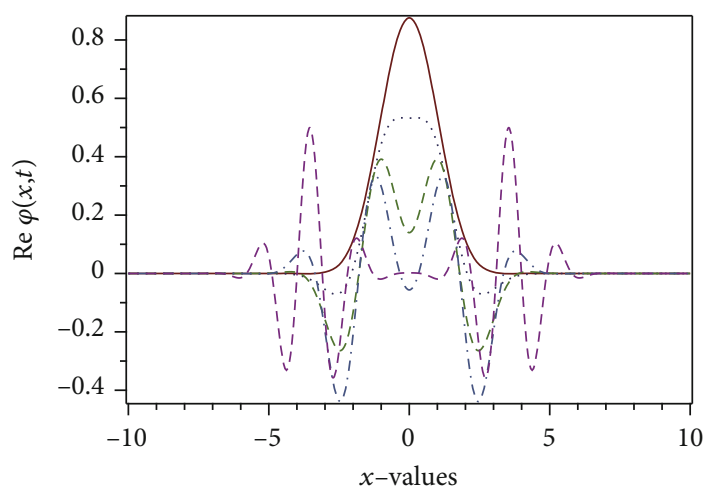

(a)

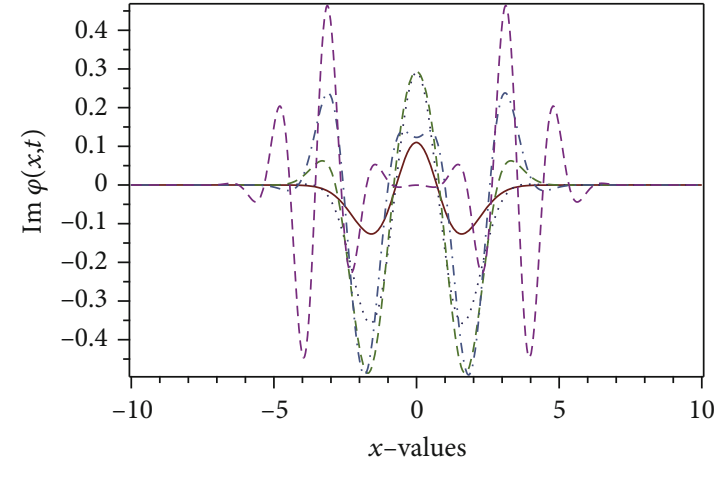

(b)

Figure 1: Real and imaginary parts of solution of (43) for $a=b=1, k=1 / 2, t=1, t=2, t=4, t=5, t=7$, and $g(x)=0$.

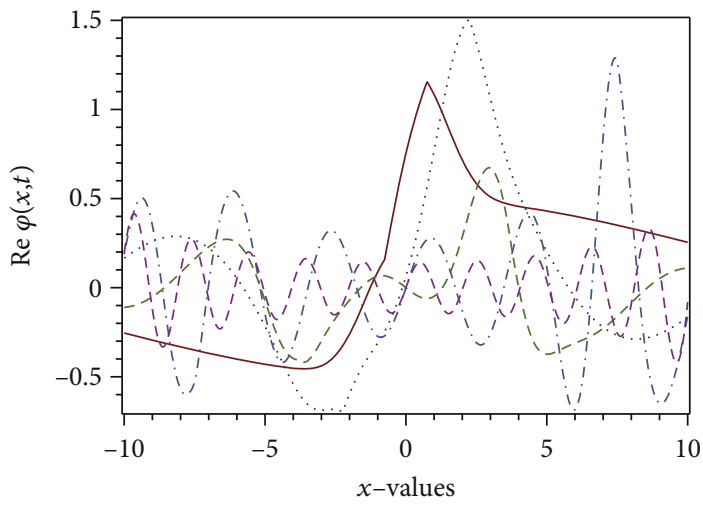

(a)

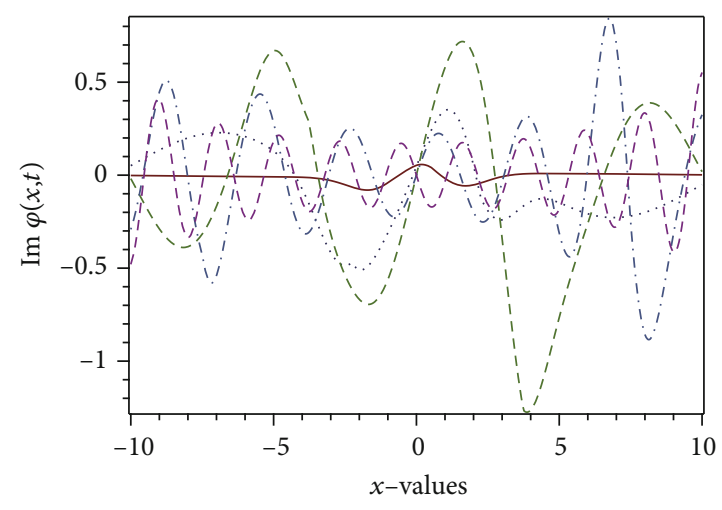

(b)

FIgURE 2: Real and imaginary parts of solution of (43) for $a=1 / 2, b=2, k=3 / 2, t=1 / 2, t=3 / 2, t=5 / 2, t=5, t=8$, and $g(x)=\operatorname{signum}(x)$.

Based on Gaussian integral and basic property of matrix parameter of the LCT, we obtain

$$
\begin{aligned}
& L_{E^{-1}}\left[e^{-\left(k^{2} / b^{2}\right) \xi^{2} t}\right]=\frac{1}{\sqrt{-2 \pi b i}} e^{-\left(a\left(4 k^{2} t-2 d b i\right) / 2 b\left(4 k^{2} t-2 d b i\right)\right) x^{2} i} e^{2 b x^{2} / 2 b\left(4 k^{2} t-2 d b i\right)} \sqrt{\frac{2 b^{2} \pi}{d b i-2 k^{2} t}} \\
& =\sqrt{\frac{b}{2 k^{2} t i+d b^{2}}} e^{\left(-4 k^{2} t a i-2 a d b+2 b / 2 b\left(4 k^{2} t-2 d b i\right)\right) x^{2}} \\
& =\sqrt{\frac{b}{2 k^{2} t i+d b^{2}}} e^{\left(-4 k^{2} t a i-2 b^{2} c / 2 b\left(4 k^{2} t-2 d b i\right)\right) x^{2}} \\
& =\sqrt{\frac{b}{2 k^{2} t i+d b^{2}}} e^{1 / 2\left(-2 k^{2} t a i-b^{2} c / 2 k^{2} b t-d b^{2} i\right) x^{2}} \text {. }
\end{aligned}
$$

Put $h(x, t)=e^{1 / 2\left(-b^{2} c-2 a k^{2} i t / 2 k^{2} b t-i b^{2} d\right) x^{2}}$. Then, we get

$$
L_{E}\left\{\sqrt{\frac{b}{2 k^{2} t i+d b^{2}}} h(x, t)\right\}=e^{-\left(k^{2} / b^{2}\right) \xi^{2} t} .
$$

From (70), we obtain

$$
\varphi(x, t)=L_{E^{-1}}\left[F_{E}(\xi) e^{-\left(k^{2} / b^{2}\right) \xi^{2} t}\right] .
$$




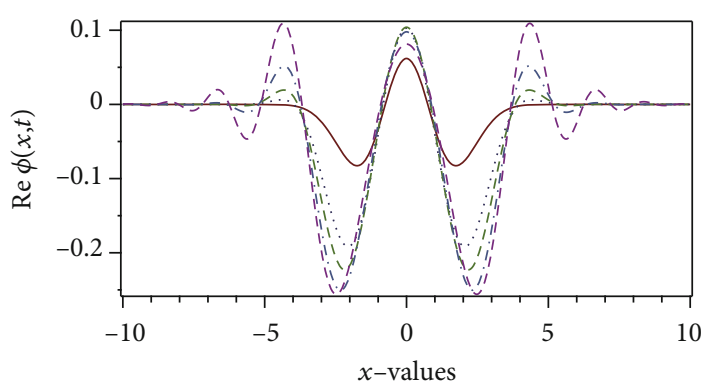

(a)

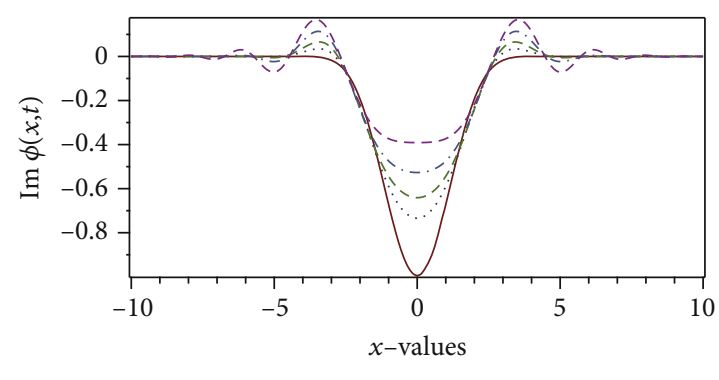

(b)

FIgURE 3: Real and imaginary parts of solution of (73) for $a=b=c=1, k=1 / 2, t=1 / 2, t=2, t=3, t=5, t=10$, and $f(x)=e^{-(1 / 2) x^{2}}$.

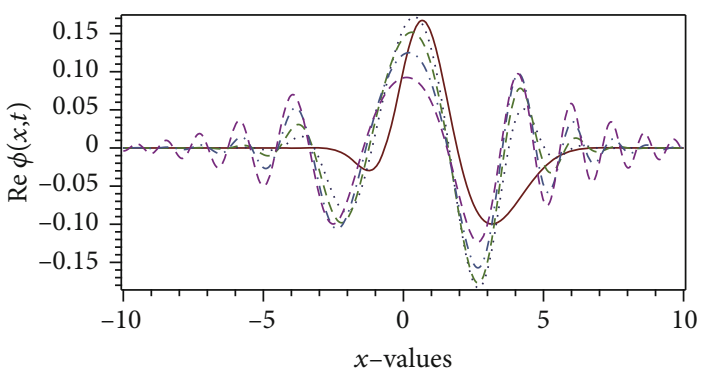

(a)

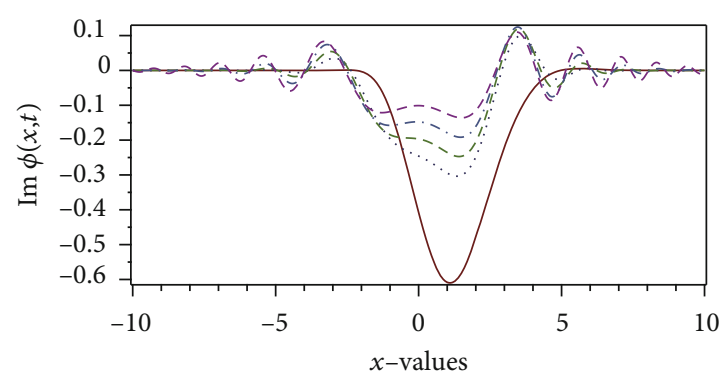

(b)

FIGURE 4: Real and imaginary parts of solution of (73) for $a=1 / 3, b=1 / 2, c=5 / 2, k=1, t=1 / 2, t=2, t=3, t=5, t=10$, and $f(x)=u(x)$.

By inserting (76) in (77), it is easy to see that

$$
\begin{aligned}
\varphi(x, t) & =L_{E^{-1}}\left[L_{E}\{f(x)\} L_{E}\left\{\sqrt{\frac{b}{2 k^{2} t i+d b^{2}}} h(x, t)\right\}\right] \\
& =\sqrt{\frac{b}{2 k^{2} t i+d b^{2}}} L_{E^{-1}}\left[L_{E}\{f(x)\} L_{E}\{h(x, t)\}\right] \\
& =\sqrt{\frac{b}{2 k^{2} t i+d b^{2}}} L_{E^{-1}}\left[\frac{1}{\sqrt{2 \pi b i}} e^{i\left(d \xi^{2} / 2 b\right)} L_{E}\{f * h\}\right] .
\end{aligned}
$$

For simplicity, let us assume $\tilde{E}=\left[\begin{array}{ll}a & b \\ c & 0\end{array}\right]$. Then, the above identity takes the form:

$$
\varphi(x, t)=\frac{\sqrt{b / 2 k^{2} t i}}{\sqrt{2 \pi b i}} L_{\tilde{A}^{-1}}\left[L_{\tilde{A}}\{f * h\}\right]
$$

Applying the convolution theorem for the LCT defined by (25), we obtain

$$
\begin{aligned}
\varphi(x, t) & =\frac{1}{\sqrt{-4 \pi k^{2} t}} L_{\tilde{A}^{-1}}\left[L_{\tilde{A}}\{f * h\}\right] \\
& =\frac{1}{\sqrt{-4 \pi k^{2} t}} \int_{\mathbb{R}} e^{(i u(u-x) a) / b} f(u) h(x-u, t) d u \\
& =\frac{1}{\sqrt{-4 \pi k^{2} t}} \int_{\mathbb{R}} e^{(i u(u-x) a) / b} f(u) e^{1 / 2\left(-b^{2} c-2 a k^{2} i t / 2 k^{2} b t\right)(x-u)^{2}} d u,
\end{aligned}
$$

as claimed in (73).
This solution of (73) with initial conditions $f(x)=$ $e^{-(1 / 2) x^{2}}$ and the Heaviside step function

$$
f(x)=u(x)= \begin{cases}0 & x<0 \\ 1 & x \geq 0\end{cases}
$$

is described in Figures 3 and 4, respectively.

Remark 17. Notice first that, when $E=\left[\begin{array}{cc}0 & 1 \\ -1 & 0\end{array}\right],(73)$ will lead to

$$
\varphi(x, t)=\frac{1}{i k \sqrt{4 \pi t}} \int_{\mathbb{R}} f(u) e^{(x-u)^{2} / 4 t k^{2}} d u
$$

The above identity is solution of the classical heat equation.

Let us consider

$$
G(x-u, t)=\frac{1}{\sqrt{-4 \pi k^{2} t}} e^{(i u(u-x) a) / b} e^{1 / 2\left(-b^{2} c-2 a k^{2} i t / 2 k^{2} b t\right)(x-u)^{2}} .
$$

Then, the solution of heat equation associated with the LCT (73) reduces to the form:

$$
\varphi(x, t)=\int_{\mathbb{R}} f(u) G(x-u, t) d u .
$$


When $f(x)=\delta(x)$, (84) becomes

$$
\varphi(x, t)=\frac{1}{\sqrt{-4 \pi k^{2} t}} e^{1 / 2\left(-b^{2} c-2 a k^{2} i t / 2 k^{2} b t\right) x^{2}} .
$$

\section{Conclusion}

We have established the solution of generalized wave equation using the ICT and obtained the solution of heat equation using the sampling formulae of band limited function related to the LCT. The solutions have been obtained by applying the properties of the LCT and the relationship between the FT and the LCT. It is found that the solutions are general form of the solutions of the classical wave and heat equations.

\section{Data Availability}

All data generated or analyzed during this study are included in this published paper.

\section{Conflicts of Interest}

The authors declare that they have no conflicts of interest.

\section{Acknowledgments}

The first author is partially supported by PDUPT from Ministry of Research, Technology and Higher Education of the Republic Indonesia. The second author is partially supported by JSPS KAKENHI (Grant Number 20K03653).

\section{References}

[1] M. Bahri, "Zulfajar and R," Ashino, Convolution and correlation theorem for linear canonical transform and properties, Information, vol. 17, no. 6B, pp. 2509-2521, 2014.

[2] M. Bahri and R. Ashino, "Convolution and correlation theorems for Wigner-Ville distribution associated with linear canonical transform," in 12th International Conference on Information Technology-New Generation, Las Vegas, NV, USA, 2015.

[3] R. Tao, Y.-L. Li, and Y. Wang, "Uncertainty principles for linear canonical transforms," IEEE Transactions on Signal Processing, vol. 57, no. 7, pp. 2856-2858, 2009.

[4] R. F. Bai, B. Z. Li, and Q.-Y. Cheng, "Wigner-Ville distribution associated with the linear canonical transform," Journal of Applied Mathematics, vol. 2012, Article ID 740161, 14 pages, 2012.

[5] D. Urynbassarova, B. Z. Li, and R. Tao, "Convolution and correlation theorems for Wigner-Ville distribution associated with the offset linear canonical transform," Optik-International Journal of Light and Electron Optics, vol. 157, pp. 455466, 2018.

[6] J. J. Healy, M. A. Kutay, H. M. Ozaktas, and J. J. Sheridon, Linear Canonical Transform: Theory and Application, vol. 198, Springer Series in Optical Sciences, 2016.

[7] S. C. Pei and S. G. Huang, "Reversible joint Hilbert and linear canonical transform without distortion," IEEE Transactions on Signal Processing, vol. 61, no. 19, pp. 4768-4781, 2013.
[8] B. Z. Li and Y. P. Shi, "Image watermarking in the linear canonical transform domain," Mathematical Problems in Engineering, vol. 2014, Article ID 645059, 9 pages, 2014.

[9] J. Biazar and R. Islam, "Solution of wave equation by Adomian decomposition method and the restrictions of the method," Applied Mathematics and Computation, vol. 149, no. 3, pp. 807-814, 2004.

[10] A. Prasad, S. Manna, A. Mahato, and V. K. Singh, “The generalized continuous wavelet transform associated with the fractional Fourier transform," Journal of Computational and Applied Mathematics, vol. 259, pp. 660-671, 2014.

[11] S. A. Collins, "Lens-system diffraction integral written in terms of matrix Optics*," Journal of the Optical Society of America, vol. 60, no. 9, pp. 1168-1177, 1970.

[12] M. Moshinsky and C. Quesne, "Linear canonical transformations and their unitary representations," Journal of Mathematical Physics, vol. 12, no. 8, pp. 1772-1780, 1971.

[13] K. B. Wolf, "Development of linear canonical transforms: a historical sketch," in Linear Canonical Transforms, Springer Series in Optical Sciences, J. Healy, K. M. Alper, H. Ozaktas, and J. Sheridan, Eds., vol. 198, Springer, New York, NY, 2016.

[14] R. Bracewell, The Fourier Transform and Its Applications, McGraw-Hill, 2000.

[15] L. Debnath and F. A. Shah, Wavelet Transform and Application, Birkhäuser, Second edition, 2015.

[16] K. Gröechenig, Foundation of Time-Frequency Analysis, Birkhäuser, Boston, 2001.

[17] K. B. Wolf, "Canonical transforms. I. Complex linear transforms," Journal of Mathematical Physics, vol. 15, no. 8, pp. 1295-1301, 1974.

[18] D. Wei, Q. Ran, and Y. Li, “A convolution and correlation theorem for the linear canonical transform and its application," Circuits, Systems, and Signal Processing, vol. 31, no. 1, pp. 301-312, 2012.

[19] D. Wei, Q. Ran, and Y. Li, "New convolution theorem for the linear canonical transform and its translation invariance property," Optik-International Journal of Light and Electron Optics, vol. 123, no. 16, pp. 1478-1481, 2012.

[20] Z. C. Zhang, "New convolution structure for the linear canonical transform and its application in filter design," Optik-International Journal of Light and Electron Optics, vol. 127, pp. 983 990, 2007.

[21] Y. Guo and B. Z. Li, "The linear canonical wavelet transform on some function spaces," International Journal of Wavelets, Multiresolution and Information Processing, vol. 16, no. 1, 2018.

[22] R. S. Pathak and A. Singh, "Mexican hat wavelet transform of distributions," Integral Transform sand Special functions, vol. 27, no. 6, pp. 468-483, 2016.

[23] A. Stern, "Sampling of linear canonical transformed signals," Signal Processing, vol. 86, no. 7, pp. 1421-1425, 2006.

[24] B. Z. Li, R. Tao, and Y. Wang, "New sampling formulae related to linear canonical transform," Signal Processing, vol. 87, pp. 5259-5263, 2007.

[25] B. Z. Li and T. Z. Xu, "Sampling in the linear canonical transform domain," Mathematical Problems in Engineering, vol. 2012, 13 pages, 2012. 